\title{
BMJ Open Tobacco use patterns and attitudes in Singapore young male adults serving military national service: a qualitative study
}

\author{
Clive Tan (D) , ${ }^{1,2}$ Lavinia Lin, ${ }^{2}$ Mervyn Lim, ${ }^{1,2}$ Seeu Kun Ong, ${ }^{1}$ Mee-Lian Wong, ${ }^{2}$ \\ Jeong Kyu Lee ${ }^{2}$
}

To cite: Tan C, Lin L, Lim M, et al. Tobacco use patterns and attitudes in Singapore young male adults serving military national service: a qualitative study. BMJ Open 2020;10:e039367. doi:10.1136/ bmjopen-2020-039367

- Prepublication history for this paper is available online To view these files, please visit the journal online (http://dx.doi. org/10.1136/bmjopen-2020039367).

Received 14 April 2020 Revised 20 July 2020 Accepted 20 August 2020

Check for updates

(c) Author(s) (or their employer(s)) 2020. Re-use permitted under CC BY-NC. No commercial re-use. See rights and permissions. Published by BMJ.

${ }^{1}$ Headquarters Medical Corps, Singapore Armed Forces Medical Corps, Singapore

${ }^{2}$ National University Singapore Saw Swee Hock School of Public Health, Singapore

Correspondence to

Dr Clive Tan;

clivetan@gmail.com

\section{ABSTRACT}

Objectives To explore tobacco use patterns and factors influencing tobacco use in young Singaporean men serving military national service.

Methods A qualitative study using in-depth telephone interviews and maximum variation sampling was conducted with 29 Singaporean men who have completed their national service in 2017-2018. Data were analysed using thematic analysis.

Results More than half (51.7\%) of the participants started smoking before age 18 , with a mean age of smoking initiation at 16 years. At the individual level, the two main contributing factors to tobacco use were smoking as a coping mechanism, and physical or psychological addiction. Financial concern was reported as the key motivation to quit smoking. At the interpersonal level, peers and superiors played an influential role in the smoking habits of the participants. When superiors took additional measures against smoking behaviours, it contributed to their decision to smoke less. At the organisation level, some unintended consequences were reported as a result of the strict tobacco control measures implemented by the military, such as the designated smoking areas within the camps become regarded as an area for socialisation.

Conclusion Informed by the Socio-Ecological Model, this study has provided insights into the multifaceted and interactive effect of individual, interpersonal and organisational factors that influence tobacco use in young men serving military national service in the Singapore and Asian context. The study insights provided an understanding of the local context before designing programmes or changing regulations to further discourage tobacco usage in the military.

\section{INTRODUCTION}

Since the 1970s, Singapore has adopted a national-level, long-term and multi-pronged approach to tobacco control. ${ }^{1}$ Currently, Singapore has the lowest smoking prevalence rates in the Southeast Asian countries, at 12\% in 2017. ${ }^{2}$ However, smoking rates among Singaporean men remain a significant issue, with a prevalence of $21.1 \%$ in $2017 .^{3}$ To
Strengths and limitations of this study

- This is the first qualitative study to explore multilevel factors that influence tobacco-use behaviour in young men serving military national service (or national servicemen) in Singapore.

- Use of purposeful random sampling stratified by selected criteria on ethnicity and educational level in the study design allowed for inclusion of diverse groups of national servicemen, to provide rich information of tobacco use.

- Data collection and analysis were underpinned by the Socio-Ecological Model as a theoretical framework.

- This study cannot be generalised to all military personnel due to the nature of qualitative design.

further reduce tobacco use in the country, the Ministry of Health (MOH) has raised the minimum legal age for the purchase, use, possession, sale and supply of tobacco products from 18 to 21 (MLA21), progressively from 2019 to $2021 .^{4}$ Coincidentally, this is the age range when all young Singaporean men serve their military national service.

In Singapore, it is mandatory for all male citizens and second-generation permanent residents to serve a 2-year period of national service (NS) as full-time national servicemen (NSFs). ${ }^{5}$ The typical age of enlistment for NS is between 18 and 21. Research into tobacco use in military populations has shown that young soldiers are at risk of smoking initiation and cigarette smoking, ${ }^{67}$ and tobacco use has been shown to increase risk of injuries and decreased fitness levels in soldiers. ${ }^{8}$ Based on a periodic health survey administered once every few years, the overall prevalence of tobacco use among NSFs in Singapore was estimated at $16.7 \%$ in 2017 . With the enactment of the MLA21, it is anticipated that a growing number of underage smokers will 
emerge in the Singapore military. Therefore, this study aimed to: (1) explore tobacco use patterns during NS among young Singaporean male adults and (2) identify the underlying factors that influence their smoking behaviours using the Socio-Ecological Model (SEM). Study findings may inform the design of policy intervention and targeted smoking prevention and cessation programmes in the military setting.

\section{METHODS}

Qualitative data were collected from March to June 2018. Participants were drawn from the database of the Singapore Armed Forces (SAF) to identify those who have completed NS in 2017-2018, and self-declared as a smoker. Purposeful random sampling ${ }^{10}$ was performed to select participants with maximum variation based on the criteria of ethnicity, educational level, and self-reported smoking status before and at the point of completion of NS. For the telephone-based interviews, all participants provided verbal consent at two points during the study period, during the recruitment call and before the conduct of the phone interview.

Participants were recruited through a collaborative effort between the SAF and the National University of Singapore (NUS). Initially, a SAF team member (ML) screened and contacted 223 eligible individuals identified from the database, explaining the purpose and procedure of the study. Of those, 64 agreed to be contacted by NUS for research purposes. Then, a NUS team member (LL) contacted 56 potential participants to conduct the telephone semi-structured interviews. Of which, 10 declined to take part and 17 were unreachable. To reduce non-response, the team made multiple attempts through repeated calling and mobile messaging at different times of the day and weekends. A total of 29 participants took part in the interviews. Telephone-based interviews were used because it permits a greater perceived level of anonymity and privacy in which the participants feel more comfortable to disclose perceived sensitive information. ${ }^{11}$

A semi-structured interview guide with open-ended questions about smoking patterns and behaviours was developed, based on the SEM. The SEM is a theory-based framework that conceptualises health behaviours as determined by an interplay between individual characteristics, interpersonal processes, organisational features and societal factors. ${ }^{12}$ This model has been widely applied in understanding complex behaviours such as tobacco use. A major strength of the model is the focus on multiple levels of influence that broadens and offers many potential points of intervention for behaviour change ${ }^{13}$ For this study, emphasis was placed on the first three levels to suggest actionable insights that potentially could be implemented in the military setting. Each phone interview lasted 40-60 $\mathrm{min}$, and a $\$ 1$ o e-voucher was provided to participants for their participation. All interviews were audio-recorded and transcribed verbatim.

\section{Patient and public involvement}

This study was designed by the authors in consultation with the leaders and researchers at the SAF's Headquarters Medical Corps, who contributed advice and helped review the research methods and findings of the study. The interview guide was pilot-tested with four NSFs who self-identified as current smokers, to gain feedback on the process and refine the interview questions.

\section{Data analysis}

Theoretical saturation was reached at 29 participants. A team of four researchers was involved in the data coding and analysis. Using the approach of thematic analysis, ${ }^{14}$ codes and subcodes was identified and subsumed under the hierarchical levels of the SEM. All transcripts were double coded and compared among coders working independently to ensure coding consistency and establish inter-rater reliability. Any discrepancies in the coding were reviewed and discussed by all team members until consensus was reached. NVivo Pro V.12 was used for the data analysis. ${ }^{15}$

\section{RESULTS}

The mean age of the participants was 22.4 years. The ethnic distribution of the participants consisted of: Malay $(44.8 \%)$, Chinese $(34.5 \%)$, Indian $(17.2 \%)$ and Others $(3.4 \%)$. Most participants had obtained a secondary education $(41.4 \%)$ or vocational institute diploma $(37.9 \%)$ at the point of enlistment for NS. Regarding smoking status, $24(82.8 \%)$ had self-reported as current smokers and $5(17.2 \%)$ as former smokers who quit smoking after completing NS. Most (84.6\%) reported that they smoked 10 or fewer cigarettes per day. Twenty-six participants $(89.7 \%)$ picked up smoking before enlistment to NS, and more than half $(51.7 \%)$ started smoking before age 18 , with a mean age of smoking initiation at 16 years of age. The most commonly reported themes for underage smoking initiation were curiosity, social influence and stress. Less than one-third (31.0\%) picked up or became a regular smoker during NS. Table 1 summarises the sociodemographic characteristics of the participants.

\section{Multiple level factors influencing tobacco use in the military setting}

Our analyses identified individual, interpersonal and organisational influences on tobacco use during NS among young Singaporean male adults. Table 2 provides the themes and codes extracted from each level.

\section{Individual level}

\section{Coping mechanism}

Participants reported that they used smoking as a coping mechanism for both stress and boredom. They highlighted that smoking was used to cope and adapt to the change of lifestyle from a civilian to a military trainee. They felt stressed from the regimentation of the military 
Table 1 Participants' demographics ( $N=29)$

\begin{tabular}{ll}
\hline Participants' demographic variables & N (\%) \\
\hline Age (in years) & \\
Mean \pm SD & $22.4 \pm 1.1$ \\
Range & $20-24$
\end{tabular}

Race

\begin{tabular}{ll} 
Chinese & $10(34.5)$ \\
Malay & $13(44.8)$ \\
Indian & $5(17.2)$ \\
Other: Filipino & $1(3.4)$ \\
Educational level at the time of enlistment & \\
Secondary education & $12(41.4)$ \\
Vocational institute & $11(37.9)$ \\
'A' levels/international baccalaureate/ & $6(20.7)$ \\
diploma & \\
\hline Tobacco use status & \\
\hline Former user & $5(17.2)$ \\
\hline Current user & $24(82.8)$ \\
Number of cigarettes smoked per day & \\
\hline 0-10 & $19(82.6)$ \\
\hline $11-30$ & $4(17.4)$ \\
Age smoked first cigarette (in years) & $16.0 \pm 2.9$ \\
\hline Mean \pm SD & $10-20$ \\
Range & \\
\hline Pre-national service smoking history & $26(89.7)$ \\
Yes & $3(10.3)$ \\
\hline No & \\
\hline
\end{tabular}

environment, and used smoking to 'cope with anxiety', 'relieve stress' and 'take our mind off the camp'. In addition, participants reported that smoking was used to 'pass time' during lull periods, as 'sometimes it's really nothing to do then we go down to smoke.' These situations occurred more commonly after they had been posted to their operational unit, possibly because they were no longer 'trainees' and given more free time in between activities.

\section{Health beliefs}

While participants were generally aware of the detriments of smoking, some had the impression that harmful effects would arise only after years of tobacco use and may not be directly applicable to them now. As one put it, "If I were to continue, [be] addicted, or go heavy, then someday it will affect me lah (the language used by participants is colloquial Singaporean English or Singlish. Singlish is a mix of words originating from several official languages and Chinese dialects, such as Hokkien and Cantonese).” The most commonly cited health consequences were lung cancer, asthma and difficulty in breathing. However, participants exhibited scepticism about the health risks as they have not yet experienced any adverse outcomes or 'seen anyone [that] has that.'Although a few reported that smoking has affected their stamina and fitness level, they believed that the body could recuperate through regular training or exercise.

\section{Quit attempt and motivations}

Participants reported that they had attempted to stop smoking at some point during NS. Quitting methods, such as cold turkey, tapering off cigarettes, distracting oneself with work, using sweets to fix oral habit fixation and by sheer willpower, were most frequently reported. A majority of them, however, relapsed back to smoking due to physical and psychological dependence. Some had experienced withdrawal symptoms, which hindered their determination in their attempts at quitting smoking. In addition, participants developed a psychological need for smoking, as it was part of their habit.'They also expressed that smoking gave them a sense of psychological comfort when they felt 'cranky,' lethargic' and 'irritated' when going too long without a cigarette.

Financial benefits associated with not smoking were reported as the strongest motivation for smoking cessation by the participants. They called smoking an 'expensive habit,' especially for the enlistees, as participants described that their allowance was low, forcing them to cut down or even quit smoking. In addition, the transition out of the military and back into civilian life prompted some participants to stop smoking, as they felt the need to save for their future: "When you are in NS, they give you pay regularly. Every month you know you will get your pay. But when you are working outside, you need to work hard for your money. So when you earn that money, you only buy the things you need." Thus, personal and future financial considerations may be a strong motivator for servicemen to cut down on tobacco use.

\section{Interpersonal level}

\section{Role of peers}

Peers played an influential role in the smoking habits of our participants. Although they noted that their smoking peers did not pressure them to smoke, they passively conformed to their peers' smoking behaviour: 'Like peers smoke, then alright lah just join them to smoke.' Participants also had a perception that 'the whole batch was all smokers,' and believed that tobacco use was 'part of the culture' in military camp. In addition, participants used smoking as a socialisation tool to build and bond relationships with their peers and superiors. As one described: "Smoking can break down barriers. Walls are broken down when you smoke and you find out more about the other party." For several participants, it is possible that they increased their cigarette intake due to the influence of peer smokers.

\section{Role of superiors and role models}

Superiors also influenced the smoking behaviours of the participants. Accounts by participants described that (non-smoking) superiors gave smoking cessation advice 
Table 2 Themes and codes identified at each level of the Socio-Ecological Model

\begin{tabular}{|c|c|c|}
\hline Level of influence & Definition of level & Theme and codes \\
\hline Individual & $\begin{array}{l}\text { Personal history and characteristics, such as } \\
\text { knowledge, attitudes and beliefs, that influence } \\
\text { tobacco-use behaviour }\end{array}$ & $\begin{array}{l}\text { Coping mechanisms } \\
\text { Transition from civilian to military life } \\
\text { Therapeutic effect of smoking } \\
\text { Health beliefs } \\
\text { Knowledge and perceived severity of } \\
\text { smoking } \\
\text { Lack of perceived susceptibility } \\
\text { Quit attempt and motivations } \\
\text { Quitting experiences } \\
\text { - Physical and psychological dependence } \\
\text { Perceived benefits of quitting smoking }\end{array}$ \\
\hline Organisational & $\begin{array}{l}\text { Policies, facilities and organisational structures } \\
\text { in the SAF camps that influence tobacco-use } \\
\text { behaviour }\end{array}$ & $\begin{array}{l}\text { SAF tobacco-control regulations } \\
\text { Common smoking rules in SAF } \\
\text { Rules about smoke breaks/ cigarette } \\
\text { possession } \\
\text { Accessibility of designated smoking areas } \\
\text { Physical distance to the yellow box } \\
\text { 'The socialisation corner' } \\
\text { Accessibility of cigarettes in camp } \\
\text { Social sources of cigarettes } \\
\text { Changes in smoking privileges from BMT to } \\
\text { operational unit } \\
\text { Strict smoking regulations during BMT } \\
\text { Relaxed smoking regulations at the } \\
\text { operational unit }\end{array}$ \\
\hline
\end{tabular}

BMT, Basic Military Taining; SAF, Singapore Armed Forces.

to their soldiers and encouraged them to quit: "My Encik [non-commissioned officer] was very supportive; he didn't allow me to smoke during my quitting periods. He helped me a lot." On the other hand, participants expressed that smoking facilitated and put them at ease in conversing with their (smoking) superiors. Participants felt that smoking together with superiors were 'bonding' and 'talking to us like friends.' As one of the participants described: "No matter what rank you are, you get to have a conversation over cigarettes."

In addition, participants reported the variability in the extent of tobacco control regulations created and enforced across companies during the Basic Military Training (BMT), which appeared to originate from commanders. They recounted that, in general, their smoke breaks or cigarette intake were limited to 'two to three sticks a day'. However, participants expressed that some superiors took additional measures against smoking behaviours among their soldiers by 'keeping the cigarette packs' and conducting 'random bag checks' to ensure there were no hidden cigarettes, and this may contribute towards their decision to smoke less. Participants further described that during smoke breaks, the sergeants "distribute our [cigarette] pack and we take one stick from there and put it back." As a result, participants felt that their cigarette intake was 'controlled' and 'restricted'. From these accounts, it appears that superiors play an important role in 
influencing the smoking habits of soldiers by their smoking behaviours and through the enforcement of smoking discipline.

\section{Role of medical officers}

Medical officers also play a significant role in smoking cessation in the military context. Participants reported that medical officers who performed medical screening for servicemen prior to completion of NS would evaluate their smoking behaviour and provide smoking cessation advice, suggesting them to quit: "They [medical officers] asked how much you smoke at that point in time, how much you smoke per day and they put a remark." However, participants felt that the medical screening process was rather procedural and ultimately had little effect on their decision to quit smoking.

\section{Organisational level}

\section{SAF tobacco-control regulations}

The SAF has implemented several smoking regulations to control cigarette consumption in the military. Cited by the participants, these rules included: no smoking in public while in uniform, banning of smoking in SAF camps except in selected designated areas and no sale of tobacco products on all SAF premises. In addition, some superior officers at the unit-level set limits on the number of daily smoke breaks and the number of cigarettes a person is allowed to bring into camp. Punishments for flouting these rules usually include stoppage of leave and the confiscation of their cigarettes. All participants were aware of these rules. Although participants described the regulations as 'restrictive', they found this approach helpful in controlling the tobacco consumption among soldiers. Some mentioned that the rules had helped cut down their intake: "Yeah tend to cut down the smoking urge. They train you to have the initiative like only smoke when you really need lah and [only] when you have the urge lah. Don't smoke for nothing. This is how I feel."

\section{Accessibility of designated smoking areas}

The concept of a designated smoking area demarcated by a yellow box as a form of tobacco-control regulation has been reported as controversial by the participants. The designated smoking area has, to some participants, provided a casual environment where "around [the smoking corner], we tend to talk more, we talk about our lives when we smoke. Because when we doing stuff we cannot talk." Thus, they regarded the designated smoking area as a place for socialisation. While these designated smoking areas should only be placed in locations that are inconvenient and out of sight, a number of participants had mentioned that these areas were sometimes sited within close proximity to their bunks or offices. Only a handful of participants complained about the long distance that they needed to travel to their designated smoking area.

\section{Accessibility of cigarettes in camp}

While cigarettes are not available for purchase on all SAF premises, they remain readily accessible. Participants usually stock up on cigarettes before booking in, or replenish their supply on 'nights out': "Before we book in, we will buy our cigarettes pack to stock up for the entire week. "Sharing cigarettes with their fellow smokers, including commanders, was also reported: "It's part of the culture. If someone wants a cigarette, it's kind of courteous to extend that help. "The participants reported that obtaining cigarettes for use in military camps was not seen as a significant barrier among the soldiers.

\section{Changes in smoking privileges from BMT to operational unit}

When progressing from BMT to the operational unit, participants recounted that it was a stage when it was easier to smoke more freely. Participants described that at their operational unit, they were no longer a trainee and had more privileges and freedom: "During BMT, they will get full control of you like things you can do or cannot do. But during unit, they are not that strict lah... more privilege was given to us. Like in between training if we have nothing to do, we can ask for a smoke break lah." As a result, most participants $(79.3 \%)$ reported that they increased their cigarette consumption as they transited from BMT to their operational unit. Some participants who held positions of authority as junior commanders, had the freedom and authority to set smoking restrictions for their subordinates. All in all, participants usually described that the smoking life in unit was 'liberating' or 'OTOT (own time own target, that is, at own pace)' which affected their smoking frequency, as they were not being monitored or tracked for their tobacco consumption.

\section{DISCUSSION}

Majority $(89.7 \%)$ of the participants started smoking before enlistment to NS, and more than half $(51.7 \%)$ started under the age of 18 (minimum legal age for smoking prior to 2019). The mean age at when our participants smoked their first cigarette was 16 years, which is consistent with the findings from the MOH's National Health Survey. ${ }^{16}$ Underage smoking and the access to cigarettes for minors remain a challenge in Singapore. ${ }^{17}$ The early and underaged initiation of smoking points to a continual need for school-based/ community-based smoking prevention and education programmes at the national level, targeted at youths to prevent them from initiating tobacco use. To address this, the Singapore Health Sciences Authority has placed strong emphasis and efforts to enforce tobacco control law in the prevention of cigarette sales to minors. Specifically, the courts had meted out heavy penalisations to vendors who flout these regulations, which included suspension or removal of their tobacco sale licences. ${ }^{18}$

Informed by the SEM, our study findings showed multifaceted effects of individual, interpersonal and organisational factors that influence tobacco use during NS among 
young Singaporean male adults. At the individual level, the two main contributing factors to tobacco use during NS were smoking as a coping mechanism, and physical or psychological addiction. Although our participants were generally aware of the harmful effects of smoking, their knowledge did not necessarily translate into practice. This could be due to the common beliefs among smokers who hold perceived control and unwarranted optimism, ${ }^{19}$ which may underestimate their smoking-related risks. ${ }^{2021}$ Such findings were also observed in our participants who were 'youthfully optimistic' about the negative health consequences of smoking. Tobacco use in the military as a way to relieve stress has been well established, ${ }^{22-24}$ and smokers were usually associated as experiencing higher levels of stress. ${ }^{22}$ Researchers have consistently recommended that military personnel need to be provided with healthier avenues and knowledge of stress-coping skills. ${ }^{24}$ Our findings further support the need of a targeted smoking cessation programme in the military setting, to equip young soldiers with appropriate stress-management strategies.

Financial concern is commonly reported by our participants as the strongest motivation for smoking cessation. In 2018, the Singapore government imposed a further $10 \%$ increase to tobacco taxes, which raised the cigarette tax incidence to $67.5 \%$ of the retail value..$^{25}$ The nation's high taxes on tobacco products and the low amount of military allowance received from NS could have made cigarettes unaffordable to the NSFs. High tobacco prices have been shown as one of the most effective tools in motivating quit attempts and reducing tobacco use among young smokers. $^{25}$

At the interpersonal level, our findings indicate that peers and superiors play a significant role in influencing smoking and quitting behaviours. Consistent with previous research, ${ }^{26-28}$ peer influence was reported as a strong factor in developing normative perceptions regarding tobacco use in which smoking was perceived as a 'social activity' and 'social norm' in the military. Our participants perceived an unrealistic high smoking norms among peers, while in fact only about one in five were smokers in the SAF. A study in the US military found that perceived smoking norms and smoking behaviours of role models were associated with the increased likelihood of smoking initiation among newly recruits. ${ }^{26}$ This suggests that non-smoking superiors may serve as an important role in promoting smoke-free lifestyle and de-normalisation of tobacco use in the military. Since 2011, the SAF has put in place a Smoking Cessation Outreach Ambassador (SCORE) Programme to address tobacco use. The mission of the SCORE is to train military personnel as outreach ambassadors and peer-counsellors, to assist peers and fellow colleagues in smoking cessation and promote tobacco prevention in camps.

While the SAF has implemented numerous tobaccocontrol measure in military camps ${ }^{29}{ }^{30}$ some unintended consequences have been reported by our participants. For example, although there are limited areas in which solders can smoke, these areas have come to being regarded as a place for socialisation. Also, while sale of tobacco products within SAF premises is prohibited, sharing of cigarettes among friends and colleagues has become an accepted social practice. Some advocates have called for a smoke-free military; however, there are limitations and constraints on adopting a true tobacco-free culture as illustrated by studies from the US military. ${ }^{23} 31$ Future studies are needed to further explore what regulations or structural barriers to tobacco use in military camp would be effective.

Our results also highlight for soldiers who have initiated smoking before joining the military, that BMT was seen as a good starting point to curb their smoking habits as trainees were placed under a heavily regimented military training environment. However, there were variations in how stringent the tobacco-control measures across different companies in BMT, which were largely leadership and personality driven. These variations have also been observed in the operational units. These variations could be reduced with organisational-level orders and guidelines regarding the implementation of antismoking regulations. This would help to standardise the norms and practices surrounding tobacco use in the military for the young soldiers and send a consistent message about tobacco control in the military camps.

The key strength of this study is the use of a comprehensive multilevel framework and qualitative design to gain insight into young Singaporean men's perceptions towards smoking and their personal experiences in NS. Participants in this study, however, may not be representative of all NSFs in Singapore. Additionally, this study focused only on the perspectives and experiences of smoking as NSFs. Further studies may involve viewpoints and experiences of the senior officers in the SAF and policymakers, to better understand the challenges and constraints related to tobacco control in the Singapore military.

\section{CONCLUSION}

This study has provided insights into the multifaceted and interactive effect of individual, interpersonal and organisational factors that influence tobacco use in young men serving military national service in the Singapore and Asian context. The insights from this study provide a useful baseline for understanding the local context and factors surrounding tobacco use in the military, and would be helpful for military leadership when considering tobacco cessation programmes or regulatory changes to further discourage tobacco usage in the military.

Acknowledgements The authors gratefully acknowledge the constructive comments offered by Professor Chia Kee Seng. We would also like to thank Low Qian Yun Fawn, Wong Tze-Jung, Tan Chiew Hoon and Dr Lim Jia Wei for their assistance in this project. A very special thanks goes to all the national servicemen who participated in the interviews.

Contributors CT, M-LW and JKL conceptualised the study design. LL, ML and SKO conducted the literature review for the study. LL conducted interviews and 
performed data analysis. LL, ML and JKL wrote the first draft of the manuscript. CT performed the final review and editing of the manuscript. All authors took an active role in developing the original ideas for the study, participant recruitment and critical revision of the manuscript for important intellectual content.

Funding This work was supported by a start-up grant from the National University of Singapore (R-608-000-179-133; Pl: JKL). This research was part of a larger project examining youth and young adult smoking in Singapore.

Competing interests None declared.

Patient consent for publication Not required.

Ethics approval The study protocol was approved by the Institutional Review Board of the Singapore Armed Force (01/18 JMC(R)).

Provenance and peer review Not commissioned; externally peer reviewed.

Data availability statement Data are available upon reasonable request. All data relevant to the study are included in the article. Anonymised transcripts will be available on request on a case-by-case basis.

Open access This is an open access article distributed in accordance with the Creative Commons Attribution Non Commercial (CC BY-NC 4.0) license, which permits others to distribute, remix, adapt, build upon this work non-commercially, and license their derivative works on different terms, provided the original work is properly cited, appropriate credit is given, any changes made indicated, and the use is non-commercial. See: http://creativecommons.org/licenses/by-nc/4.0/.

\section{ORCID iD}

Clive Tan http://orcid.org/0000-0001-9573-2068

\section{REFERENCES}

1 Ministry of Health. Public consultation paper on proposed TobaccoControl measures in Singapore. Singapore, 2018.

2 Tan Y, Dorotheo U. The tobacco control atlas: ASEAN region. Fourth edn. Bangkok, Thailand, 2018.

3 Ministry of Health. Executive summary on national population health survey 2016/17. Singapore, 2018.

4 Ministry of Health. Minimum legal age for tobacco raised to 19 years old. Singapore, 2018.

5 Singapore Statutes Online. Chapter 93. In: Enlistment act. Singapore, 1970.

6 Talcott GW, Ebbert JO, Klesges RC, et al. Tobacco research in the military: reflections on 20 years of research in the United States air force. Mil Med 2015;180:848-50.

7 Chu N-F, Lin F-H, Wu Y-C. Prevalence and trends of cigarette smoking among military personnel in Taiwan: results of 10-year anti-smoking health promotion programs in military. Mil Med 2017;182:e1933-7.

8 Bedno SA, Jackson R, Feng X, et al. Meta-Analysis of cigarette smoking and musculoskeletal injuries in military training. Med SCi Sports Exerc 2017;49:2191-7.

9 Feinberg JH, Ryan MAK, Johns M, et al. Smoking cessation and improvement in physical performance among young men. Mil Med 2015;180:343-9.
10 Palinkas LA, Horwitz SM, Green CA, et al. Purposeful sampling for qualitative data collection and analysis in mixed method implementation research. Adm Policy Ment Health 2015;42:533-44.

11 Oltmann SM. Qualitative interviews: a methodological discussion of the interviewer and respondent contexts. Forum Qual Sozialforsch 2016.

12 McLeroy KR, Bibeau D, Steckler A, et al. An ecological perspective on health promotion programs. Health Educ Q 1988;15:351-77.

13 Glanz K, Rimer BK, Viswanath K. Health Behavior: Theory, Research, and Practice, 2015.

14 Braun V, Clarke V. Using thematic analysis in psychology. Qual Res Psychol 2006;3:77-101.

15 QSR International Pty Ltd. NVivo qualitative data analysis software, 2018.

16 Ministry of Health. National health survey 2010. Singapore, 2010.

17 Paulo DA. Underage smokers: the ease of getting cigarettes put to the test. Channel News Asia, 2017.

18 Health Sciences Authority Singapore. Hsa Revokes Licence of Retailer who sold tobacco to Under-Aged person in school uniform, suspends four others. Singapore, 2019.

19 Masiero M, Lucchiari C, Pravettoni G. Personal fable: optimistic bias in cigarette smokers. Int J High Risk Behav Addict 2015;4:e20939.

20 Weinstein ND, Marcus SE, Moser RP. Smokers' unrealistic optimism about their risk. Tob Control 2005;14:55-9.

21 Dillard AJ, McCaul KD, Klein WMP. Unrealistic optimism in smokers: implications for smoking myth endorsement and self-protective motivation. J Health Commun 2006;11 Suppl 1:93-102.

22 Stein RJ, Pyle SA, Haddock CK, et al. Reported stress and its relationship to tobacco use among U.S. military personnel. Mil Med 2008;173:271-7.

23 Smith EA, Grundy Q, Malone RE. "It's not a priority when we're in combat": public health professionals and military tobacco control policy. Am J Public Health 2015;105:660-4.

24 Smith EA, Malone RE. Mediatory myths in the U.S. military: tobacco use as "stress relief". Am J Health Promot 2014;29:115-22.

25 Lee JK, Lin L, Lim MJR, et al. National tobacco control policies from the perspectives of Singapore young male adults. J Psychoactive Drugs 2020;52:5-12.

26 Green KJ, Hunter CM, Bray RM, et al. Peer and role model influences for cigarette smoking in a young adult military population. Nicotine Tob Res 2008;10:1533-41.

27 Tai Z, Tao S-P, Hung Y-J. Peer influence and smoking relapse among active-duty military personnel in Taiwan. Tob Control 2011;20:444-5.

28 Patten C, Wang X-Q, Ebbert JO, et al. Influence of gender and peer tobacco use on tobacco use intentions after a period of involuntary tobacco abstinence among U.S. air force trainees. Prev Med Rep 2019;13:270-6.

29 Goh SM. SAF's anti-smoking rules in line with national policies. The Straits Times, 2019.

$30 \mathrm{CW}$ A. MINDEF reviewing therapies to help personnel stub out. The Straits Times, 2018.

31 Smith EA, Malone RE. Why strong tobacco control measures "can't" be implemented in the U.S. Military: a qualitative analysis. Mil Med 2012;177:1202-7. 